\title{
ON THE HARNACK CONSTANT AND THE BOUNDARY BEHAVIOR OF HARNACK FUNCTIONS
}

\author{
MATTI VUORINEN
}

\section{Introduction}

Let $G$ be a domain in $R^{n}, n \geqq 2, G \neq R^{n}, R_{+}=\left\{x \in R^{1}: x>0\right\}$ and let $u: G \rightarrow$ $\rightarrow R_{+} \cup\{0\}$ be a continuous function. Then $u$ is said to satisfy the Harnack inequality or to be a Harnack function if there are constants $\lambda \in(0,1)$ and $C_{\lambda} \geqq 1$ such that the inequality

$$
\max _{\bar{B}^{n}(x, \lambda r)} u(z) \leqq C_{\lambda} \min _{\bar{B}^{n}(x, \lambda r)} u(z)
$$

holds whenever $B^{n}(x, r) \subset G$. Let now $F \neq \emptyset$ be a compact set in $G$. It is well-known that there exists a smallest constant $C(F) \geqq C_{\lambda}$ depending only on $n, \lambda, C_{\lambda}$, and $F$ (cf. [7, p. 16]) such that for all $u$ satisfying (1.1)

$$
\max _{F} u(z) \leqq C(F) \min _{F} u(z) .
$$

The number $C(F)$ will be termed the Harnack constant of $F$. The class of functions satisfying (1.1) is wide: it includes non-negative harmonic functions as well as nonnegative solutions of some elliptic equations (cf. [7]). In particular, the partial differential equations associated with quasiregular mappings belong to this family of elliptic equations ([9], [15]).

The purpose of the present paper is to study the boundary behavior of Harnack functions at an individual boundary point. The main tool we will use is a sharp upper bound of the Harnack constant proved in Section 3. Some related upper bounds in the case of harmonic functions were proved by Köhn [11] and Beleckaja and Landkof [3]. The upper bound of $C(F)$ in Section 3 depends on $n, \lambda, C_{\lambda}$, and the quasihyperbolic diameter of $F$. Preliminary results about the quasihyperbolic metric are given in Section 2. The main results are presented in Sections 4 and 6. In Section 4 we assume that a Harnack function of $R_{+}^{n}$ tends to 0 either through a sequence of points converging to the origin or through a curve terminating at the origin, and wish to prove that the function has an angular limit 0 at the origin. Several examples are constructed to show that the results established are the best possible and to illuminate various phenomena in the boundary behavior of Harnack functions. In Section 5 we study functions satisfying the following uniform Harnack inequality: (1.1) holds for each 
$\lambda \in(0,1)$ with $C_{\lambda \rightarrow 1}$ as $\lambda \rightarrow 0$. These functions will be characterized by means of uniform continuity with respect to the quasihyperbolic metric. The results of Section 4 do not hold if the sequential or asymptotic limits considered equal $\alpha>0$ instead of being 0 , not even for functions satisfying the uniform Harnack inequality. Motivated by this observation we shall discuss the notion of an approximate limit in Section 6 and prove that a function satisfying the uniform Harnack inequality and having an approximate limit $\alpha \geqq 0$ has an angular limit $\alpha$.

Some results in this paper, including Theorems 4.5 and 6.13 , although analogous with known boundary properties of some other classes of functions (cf. [4], [13], [17]), are perhaps new even for harmonic functions in the plane. A Harnack function need not satisfy a maximum principle, i.e., it need not be monotone in the sense of Lebesgue. In [19] some results related to this paper were proved in the case of monotone $\mathrm{ACL}^{n}$ functions with a finite Dirichlet integral.

\section{Preliminary results}

2.1. For $x \in R^{n}, n \geqq 2$, and $r>0$ let $B^{n}(x, r)=\left\{z \in R^{n}:|z-x|<r\right\}, S^{n-1}(x, r)=$ $\partial B^{n}(x, r), B^{n}(r)=B^{n}(0, r), S^{n-1}(r)=\partial B^{n}(r), B^{n}=B(1)$, and $S^{n-1}=\partial B^{n}$. The standard coordinate unit vectors are $e_{1}, \ldots, e_{n}$. If $A \subset R^{n}$, let $A_{+}=\left\{x=\left(x_{1}, \ldots, x_{n}\right) \in A\right.$ : $\left.x_{n}>0\right\}$. The hyperbolic metric $\varrho$ in $R_{+}^{n}$ is defined by the element of length $d \varrho=|d x| / x_{n}$. If $x \in R_{+}^{n}$ and $M>0$, we write $D(x, M)=\left\{z \in R_{+}^{n}: \varrho(z, x)<M\right\}$. A basic fact is that the hyperbolic balls are euclidean ones, and for instance

$$
D\left(t e_{n}, M\right)=B^{n}\left((t \cosh M) e_{n}, t \sinh M\right)
$$

for $t>0$. Let $x, y \in R_{+}^{n}$. As in the case $n=2$ [2, Theorem 6.3.1 (ii)], we have the formula

$$
\cosh \varrho(x, y)=1+\frac{|x-y|^{2}}{2 x_{n} y_{n}} .
$$

The geodesic curve joining $a \in R_{+}^{n}$ to $b \in R_{+}^{n}$ lies on an circular arc through $a$ and $b$, perpendicular to $\partial R_{+}^{n}$. Making use of this fact one calculates for $\alpha \in(0, \pi / 2)$

$$
\varrho\left(e_{n},(\cos \alpha) e_{1}+(\sin \alpha) e_{n}\right)=-\log \tan \frac{\alpha}{2} .
$$

2.5. Quasihyperbolic metric. Let $G \subset R^{n}, G \neq R^{n}$, be a domain and $a, b \in G$. The quasihyperbolic distance between $a$ and $b$ is defined by

$$
k_{G}(a, b)=\inf _{\gamma} \int_{\gamma} \operatorname{dist}(x, \partial G)^{-1} d s,
$$

where the infimum is taken over all rectifiable curves $\gamma \subset G$ with $a \in \gamma, b \in \gamma$ (Gehring-Palka [6, p. 173], Gold'šmidt-Suvorov [8, p. 61]). Set $k_{G}(A)=$ $\sup \left\{k_{G}(x, y): x, y \in A\right\}$ for $A \subset G$ and $d(z)=\operatorname{dist}(z, \partial G)$ for $z \in G$. In [6, 2.1] 
it was proved that

$$
\begin{gathered}
k_{G}(x, y) \geqq\left|\log \frac{d(y)}{d(x)}\right|, \\
k_{G}(x, y) \geqq \log \left(1+\frac{|x-y|}{d(x)}\right),
\end{gathered}
$$

for $x, y \in G$. Here equality holds if $G=R_{+}^{n}$ and $x=e_{n}, y=t e_{n}, t>1$, and hence the bounds are sharp. For $x \in G$ and $y \in B^{n}(x, d(x))$ we have $[18,2.11]$

$$
k_{G}(x, y) \leqq \log \left(1+\frac{|x-y|}{d(x)-|x-y|}\right) .
$$

2.8. Remarks. (1) If $G=R_{+}^{n}$, the quasihyperbolic metric is the same as the ordinary hyperbolic metric $\varrho$. If $G=B^{n}$, then $\varrho / 2 \leqq k_{B^{n}} \leqq \varrho$, where $\varrho$ is the ordinary hyperbolic metric of $B^{n}, d \varrho=2|d x| /\left(1-|x|^{2}\right)$.

(2) If $\gamma$ is a curve in $G$, then $k_{G}(\gamma) \leqq s(\gamma) / d(\gamma, \partial G)$, where $s(\gamma)$ is the euclidean length of $\gamma$.

(3) If $F \subset G$ is compact and connected, $k_{G}(F)$ has an upper bound in terms of $d(F) / d(F, \partial G)$ and the dimension $n$ (cf. $[18,2.18],[16,6.9])$.

2.9. ( $a, b, \lambda$ )-admissible families. Let $G \subset R^{n}, G \neq R^{n}$, be a domain and let $a, b \in G, \lambda \in(0,1)$. A family $\left\{B^{n}\left(x_{i}, r_{i}\right): i=1, \ldots, p\right\}$ of balls in $G$ is said to be $(a, b, \lambda)$ admissible $[18,2.13]$ if the following conditions are satisfied:

$$
\begin{gathered}
a \in \bar{B}^{n}\left(x_{1}, \lambda r_{1}\right), b \in \bar{B}^{n}\left(x_{p}, \lambda r_{p}\right), \\
\bar{B}^{n}\left(x_{i}, \lambda r_{i}\right) \cap \bar{B}^{n}\left(x_{i+1}, \lambda r_{i+1}\right) \neq \emptyset \text { for } i=1, \ldots, p-1 .
\end{gathered}
$$

The next lemma gives estimates for the number of balls in an $(a, b, \lambda)$-admissible family, and it is a modification of the chaining lemma in [18, 2.14].

2.10. Lemma. Let $a, b \in G$ and $\lambda \in(0,1)$.

(1) Every $(a, b, \lambda)$-admissible family contains at least $k_{G}(a, b) /(2 \log (1 /(1-i))$. balls.

(2) There exists an $(a, b, \lambda)$-admissible family with at most $1+k_{G}(a, b) / c, c=$ $\log ((1+3 \lambda) /(1+\lambda))$, balls.

Proof. Part (1) was proved in $[18,2.14]$. To prove (2), let $\gamma$ be a rectifiable geodesic curve of the quasihyperbolic metric with $a, b \in \gamma[5$, Lemma 1]. Let $\mu \in(0,1)$. Set $x_{1}=a$ and let $x_{2}, \ldots, x_{p}, x_{p+1}=b$ be points on $\gamma$ such that

$$
\left|x_{j}-x_{j+1}\right|=\mu d\left(x_{j}\right), j=1, \ldots, p-1,\left|x_{p}-x_{p+1}\right| \leqq \mu d\left(x_{p}\right),
$$

and such that $x_{j+1}, 1 \leqq j \leqq p-1$, is the last point in $\partial B^{n}\left(x_{j}, \mu d\left(x_{j}\right)\right) \cap \gamma$ when we traverse from $a$ to $b$ along $\gamma$. Define $z_{j}=\left(x_{j}+x_{j+1}\right) / 2, \quad r_{j}=\left|x_{j}-x_{j+1}\right| / 2, D_{j}=$ 
$B^{n}\left(z_{j}, d\left(z_{j}\right)\right), j=1, \ldots, p$. We want to find a condition on $\mu$ under which $\left\{D_{j}: j=1, \ldots, p\right\}$, is $(a, b, \lambda)$-admissible. A sufficient condition is

$$
\bar{B}^{n}\left(z_{j}, \lambda d\left(z_{j}\right)\right) \cap \bar{B}^{n}\left(z_{j+1}, \lambda d\left(z_{j+1}\right)\right) \neq \emptyset,
$$

which is satisfied, provided that

$$
\lambda d\left(z_{j}\right) \geqq r_{j}=\mu d\left(x_{j}\right) / 2 .
$$

On the other hand, $d\left(z_{j}\right) \geqq d\left(x_{j}\right)(1-\mu / 2)$. Thus a sufficient condition is $\lambda(1-\mu / 2) \geqq$ $\geqq \mu / 2$. We choose $\mu=2 \lambda /(1+\lambda)$. By (2.6) we get

$$
k_{G}\left(x_{j}, x_{j+1}\right) \geqq \log \left(1+\frac{\left|x_{j}-x_{j+1}\right|}{d\left(x_{j}\right)}\right)=\log \left(1+\frac{\mu d\left(x_{j}\right)}{d\left(x_{j}\right)}\right)=\log \frac{1+3 \lambda}{1+\lambda} .
$$

We now obtain the estimate

$$
k_{G}(a, b)=\int_{\gamma} \frac{d s}{d(x)} \geqq \sum_{j=1}^{p-1} k_{G}\left(x_{j}, x_{j+1}\right) \geqq(p-1) \log \frac{1+3 \lambda}{1+\lambda} .
$$

The desired upper bound for $p$ follows from this estimate.

2.11. Remark. If $a, b \in G=R_{+}^{n}$, then the estimates in Lemma 2.10 can be improved as we will now show. By virtue of (2.2) we have for $x=\left(x_{1}, \ldots, x_{n}\right) \in R_{+}^{n}$

$$
\varrho\left(B^{n}\left(x, \lambda x_{n}\right)\right)=\log \frac{1+\lambda}{1-\lambda} .
$$

By the proof of $[18,2.14$ (1)] it is clear that the lower bound 2.10 (1) can be replaced by $\varrho(a, b) / \log ((1+\lambda) /(1-\lambda))$. Denote by $J[u, v]$ the geodesic segment of the hyperbolic metric of $R_{+}^{n}$ joining $u \in R_{+}^{n}$ to $v \in R_{+}^{n}$. For the upper bound choose $x_{1}=a, x_{2}, \ldots, x_{p}, x_{p+1}=b$ on $J[a, b]$ such that

$$
\begin{gathered}
\varrho\left(x_{1}, x_{j}\right)=(j-1) \log \frac{1+\lambda}{1-\lambda}, \quad j=2, \ldots, p, \\
\varrho\left(x_{p}, x_{p+1}\right) \leqq \log \frac{1+i}{1-i} .
\end{gathered}
$$

Let $z_{j} \in J\left[x_{j}, x_{j+1}\right], j=1, \ldots, p$, be such that

$$
\varrho\left(x_{1}, z_{j}\right)=\left(j-\frac{1}{2}\right) \log \frac{1+\lambda}{1-\lambda} .
$$

Then $\left\{B^{n}\left(y_{j}, y_{j n}\right): j=1, \ldots, p\right\}$ is $(a, b, \lambda)$-admissible. Here $y_{j n}$ is the $n$-th coordinate of $y_{j}$ and $y_{j}$ is such that

(cf. (2.2)).

$$
B^{n}\left(y_{j}, \lambda y_{j n}\right)=D\left(z_{j}, \frac{1}{2} \log \frac{1+\lambda}{1-\lambda}\right)
$$


It follows that

$$
p \leqq 1+\varrho(a, b) / \log \frac{1+\lambda}{1-\lambda},
$$

which is better than the bound given by $2.10(2)$.

\section{An upper bound for the Harnack constant}

In this section we shall apply Lemma 2.10 to produce an upper bound of the Harnack constant. We also give several examples of Harnack functions, and, in particular, introduce a Harnack function $u_{F}$ associated with a relatively closed set $F \subset G$. With the aid of the functions $u_{F}$ we show that the upper bound of the Harnack constant is sharp for $G=R_{+}^{n}$. We first give some examples of Harnack functions.

3.1. Examples. (1) A continuous function $u: G \rightarrow R_{+}$with $0<m \leqq u(x) \leqq M<\infty$ for all $x \in G$ satisfies (1.1) with $C_{\lambda}=M / m$.

(2) Let $u: G \rightarrow R_{+}$be defined by $u(x)=A d(x, \partial G)^{\alpha}, A>0, \alpha \neq 0$. Because $(1-\lambda) d(y) \leqq d(x) \leqq(1+\lambda) d(y)$ for $x \in \bar{B}^{n}(y, \lambda d(y)), u$ satisfies (1.1) with $C_{\lambda}=$ $((1+\lambda) /(1-\lambda))^{|\alpha|}$.

(3) Let $E \subset G$ be closed. Then $v: G \rightarrow R_{+}, v(x)=d(x) /(d(x)+d(x, E))$ satisfies (1.1) with $C_{\lambda}=(1+\lambda) /(1-\lambda)$.

(4) Non-negative harmonic functions satisfy (1.1) with a constant $C_{2}$, such that $C_{\lambda \rightarrow 1}$ as $\lambda \rightarrow 0$ (cf. [7, p. 28, 2.6]).

3.2. Lemma. Let $u: G \rightarrow R_{+}$and $v: G \rightarrow R_{+}$be continuous functions. If $u$ satisfies (1.1), then also (1) $\alpha u^{p}+\beta(\alpha, \beta>0, p \in R)$ and (2) $u /(u+1)$ satisfy it. (3) If $0<u(x)<1$ for $x \in G$ and $u$ satisfies (1.1), then also $-\log u$ does. (4) If there exists $\tau \in(0,1)$ such that $\tau \leqq u(x) / v(x) \leqq 1 / \tau$ for $x \in G$ and $u$ satisfies $(1.1)$, then also $v$ satisfies it. If both $u$ and $v$ satisfy (1.1), then (5) $u v,(6) \max (u, v), \min (u, v),(7) u+v$ satisfy it.

The proof is a simple verification left to the reader.

3.3. Lemma. Suppose that $u: G \rightarrow R_{+} \cup\{0\}$ satisfies (1.1) and $F \subset G$ is compact. Then

$$
\max _{F} u(z) \leqq C(F) \min _{F} u(z) ; \quad C(F) \leqq C_{\lambda}^{1+t},
$$

where $t=k_{G}(F) / \log ((1+3 \lambda) /(1+\lambda))$. In particular, $u=0$ if $u\left(x_{0}\right)=0$ for some $x_{0} \in G$. If $G=R_{+}^{n}$, one may choose $t=\varrho(F) / \log ((1+\lambda) /(1-\lambda))$.

Proof. Let $a$ and $b$ be the points in $F$ where $u$ attains its greatest and least value in $F$, respectively. By 2.10 and (2.13) there exists an $(a, b, \lambda)$-admissible family 
$\left\{B_{j}: j=1, \ldots, p\right\}$, where $B_{j}=B^{n}\left(x_{j}, r_{j}\right)$ and $p \leqq 1+t$. Fix $x_{j} \in \bar{B}^{n}\left(x_{j}, \lambda r_{j}\right) \cap \bar{B}^{n}\left(x_{j+1}\right.$, $\left.\lambda r_{j+1}\right)$. By (1.1) we get

$$
u(a) \leqq \max _{E_{1}} u(z) \leqq C_{\lambda} \min _{E_{1}} u(z) \leqq C_{\lambda} u\left(x_{1}\right) \leqq C_{\lambda} \max _{E_{2}} u(z) \leqq C_{\lambda}^{2} \min _{E_{2}} u(z),
$$

where $E_{j}=\bar{B}^{n}\left(x_{j}, \lambda r_{j}\right)$. This yields the desired bound.

3.4. Corollary. Let $u: G \rightarrow R_{+} \cup\{0\}$ satisfy (1.1). Then

$$
C_{\lambda}^{-1-t} u(y) \leqq u(x) \leqq C_{\lambda}^{1+t} u(y)
$$

for all $x, y \in G$ where $t=k_{G}(x, y) / \log ((1+3 \lambda) /(1+\lambda))$. For $G=R_{+}^{n}, \quad t=\varrho(x, y) /$ $\log ((1+\lambda) /(1-\lambda))$.

3.5. Remark. An estimate related to 3.4 was obtained by Beleckaja and Landkof [3] in the case of harmonic functions. The upper bound in [3] is given in terms of the numbers

$$
\begin{gathered}
\beta(x, y)=\inf \{s(\gamma) / d(\gamma, \partial G)\}, \\
\beta(F)=\sup \{\beta(x, y) ; x, y \in F\},
\end{gathered}
$$

where the infimum is taken over all rectifiable curves $\gamma \subset G$. Let now $G=R_{+}^{n}$, $F_{t}=\left\{e_{n}, t e_{n}\right\}, t \in(0,1)$. The upper bound $\log C\left(F_{t}\right) \leqq n \beta\left(F_{t}\right)=n(1-t) / t$ is obtainable from [3] while Lemma 3.3 yields $\log C\left(F_{t}\right) \leqq d\left(\lambda, C_{\lambda}\right)(\log (1 / t)+1)$ (cf. (2.6)). For $n=2$ there are sharp results due to Köhn [11] in the case of harmonic functions.

We shall now introduce a convenient way of constructing Harnack functions with the aid of the quasihyperbolic metric. The first application of this construction will produce a lower bound of the Harnack constant associated with the class of functions satisfying (1.1) for fixed $\lambda \in(0,1)$ and $C_{\lambda} \geqq 1$.

3.6. The function $u_{F}$. Let $F \subset G$ be a closed set, $F \neq \emptyset$. For $x \in G$ and $t \in R$ set

$$
u_{F}^{t}(x)=\exp \left(-t k_{G}(F, x)\right), u_{F}(x)=u_{F}^{1}(x) .
$$

Clearly $u_{F}(x)=1$ for $x \in F$. If $F \subset G$ is compact, then $u_{F}(x) \rightarrow 0$ as $x \rightarrow \partial G$.

3.8. Lemma. The function $u_{F}^{t}: G \rightarrow R_{+}$satisfies (1.1) with $C_{\lambda}=(1-\lambda)^{-2|t|}$. If $G=R_{+}^{n}$, we can choose $C_{\lambda}=((1+\lambda) /(1-\lambda))^{|t|}$.

Proof. Fix $\lambda \in(0,1)$. Let $x \in G$ and $r>0$ be such that $B^{n}(x, r) \subset G$. Write $m=\inf \left\{k_{G}(z, F):|z-x|<r\right\}$ and $M=\sup \left\{k_{G}(z, F):|z-x|<r\right\}$. Clearly

$$
M \leqq m+k_{G}\left(B^{n}(x, \lambda r)\right) \leqq m+k_{G}\left(B^{n}(x, \lambda d(x))\right) \leqq m+2 \log \frac{1}{1-\lambda},
$$

where in the last step we have applied (2.7). The proof is now an easy verification. For the case $G=R_{+}^{n}$ use (2.12) in place of (2.7).

3.9. Example. Let $\lambda \in(0,1)$ and $C_{\lambda} \geqq 1$ be given and let $U=\left\{u: G \rightarrow R_{+}: u\right.$ satisfies (1.1) with the given numbers\}. Fix $x_{0} \in G$. Then $u_{x_{0}}^{A} \in U$ by 3.8 when $A=$ 
$\left(\log C_{\lambda}\right) /(2 \log (1 /(1-\lambda)))\left(A=\left(\log C_{\lambda}\right) / \log ((1+\lambda) /(1-\lambda))\right.$ for $\left.G=R_{+}^{n}\right)$. ' For $y \in G$ we have

$$
u_{x_{0}}^{A}\left(x_{0}\right) / u_{x_{0}}^{A}(y)=\exp \left\{A k_{G}\left(x_{0}, y\right)\right\} .
$$

Corollary 3.4 yields the following upper bound for all $v \in U$

$$
v\left(x_{0}\right) / v(y) \leqq C_{\lambda}^{1+t} ; \quad t=k_{G}\left(x_{0}, y\right) / \log \frac{1+3 \lambda}{1+\lambda},
$$

$\left(t=\varrho\left(x_{0}, y\right) / \log ((1+\lambda) /(1-\lambda))\right.$ for $\left.G=R_{+}^{n}\right)$. In conclusion, from (3.10) we get a lower bound of the Harnack constant associated with the set $F=\left\{x_{0}, y\right\}$ and with the class of functions $U$ while (3.11) yields the corresponding upper bound. Note that for $G=R_{+}^{n}$ both these numbers are of the same order of magnitude when $x_{0}$ is kept fixed and $y$ varies.

3.12. Remarks. It is well-known that if $F$ is connected, then $C(F)$ has an upper bound in terms of $n, C_{\lambda}, \lambda$, and the ratio $d(F) / d(F, \partial G)$ (cf. [10, p. 263]). This fact also follows from Lemma 3.3 and Remark 2.8 (3). Let us now show that the situation is different for non-connected $F$. For this purpose consider $u: R^{2} \backslash X_{+} \rightarrow(0,2 \pi)$, $u(z)=\arg z$, where $X_{+}$is the non-negative real axis. Then $u$ satisfies (1.1) with $C_{\lambda}=$ $((1+\lambda) /(1-\lambda))$. For $\varphi \in(0, \pi / 2)$ let $F_{\varphi}=\left\{x_{\varphi}, x_{2 \pi-\varphi}\right\}$, where $x_{\varphi}=(\cos \varphi, \sin \varphi)$. We have a lower bound for $C\left(F_{\varphi}\right)$

$$
C\left(F_{\varphi}\right) \geqq \frac{u\left(x_{2 \pi-\varphi}\right)}{u\left(x_{\varphi}\right)}=\frac{2 \pi-\varphi}{\varphi} \geqq \frac{5}{\varphi} \quad \text { for } \quad \varphi \in(0,1 / 2) .
$$

On the other hand, $d\left(F_{\varphi}\right) / d\left(F_{\varphi}, \partial\left(R^{2} \backslash X_{+}\right)\right)=2$ for $\varphi \in(0,1 / 2)$. In view of the above lower bound we see that it is not possible to estimate $C\left(F_{\varphi}\right)$ from above in terms of $d\left(F_{\varphi}\right) / d\left(F_{\varphi}, \partial\left(R^{2} \backslash X_{+}\right)\right)$. Let us finally introduce the following condition under which such an estimate is possible. There exists a constant $D \geqq 1$ such that any two points $x, y \in G$ can be joined by a curve $\gamma \subset G$ with the properties (i) $s(\gamma) \leqq D|x-y|$ and (ii) $d(\gamma, \partial G) \geqq D^{-1} \min \{d(x), d(y)\}$. All convex domains satisfy this condition with $D=1$. An important class of domains satisfying the condition is the class of uniform domains (cf. [5]).

\section{On angular limits of Harnack functions}

In the remaining part of the paper we will study the boundary behavior of Harnack functions and of other related classes of functions. We shall illuminate the results by constructing several examples of the possible behavior of the functions at boundary with the aid of the functions $u_{F}$ introduced in Section 3 . We usually confine our attention to the case of functions defined in $R_{+}^{n}$, although sometimes the results have a counterpart in the case of more general domains. 
Several results in the present section have their counterparts for other classes of functions (cf. [1], [17]-[19]). The first result follows from 3.4.

4.1. Corollary. Let $u: R_{+}^{n} \rightarrow R_{+}$be a Harnack function, $b_{k} \in R_{+}^{n}, \quad b_{k} \rightarrow 0$, $u\left(b_{k}\right) \rightarrow 0$, and $M>0$. Then $u(x) \rightarrow 0$ as $x \rightarrow 0$ and $x \in \cup D\left(b_{k}, M\right)$.

4.2. Example. As the function $u: R_{+}^{2} \rightarrow R_{+}, u(z)=\arg z \in(0, \pi)$, shows, the condition $u\left(b_{k}\right) \rightarrow 0$ in 4.1 cannot be replaced by $u\left(b_{k}\right) \rightarrow \beta, \beta>0$.

For $\varphi \in(0, \pi / 2)$ let $C(\varphi)=\left\{z \in R_{+}^{n}:\left(z \mid e_{n}\right)>|z| \cos \varphi\right\}$, where $(x \mid y)=\sum_{i=1}^{n} x_{i} y_{i}$. A function $u: R_{+}^{n} \rightarrow R$ is said to have an angular limit $\alpha$ at 0 if for each $\varphi \in(0, \pi / 2)$ $u(x) \rightarrow \alpha$ as $x \rightarrow 0, x \in C(\varphi)$. The function $u$ is said to have an asymptotic value $\alpha$ at 0 if there exists a curve $\gamma:[0,1) \rightarrow R_{+}^{n}$ such that $\gamma(t) \rightarrow 0$ and $u(\gamma(t)) \rightarrow \alpha$ as $t \rightarrow 1$. A set $E \subset R_{+}^{n}$ with $0 \in \bar{E}$ is said to be non-tangential at 0 if $E \subset C(\varphi)$ for some $\varphi \in(0, \pi / 2)$. Otherwise $E$ is said to be tangential at 0 .

4.3. Theorem. Let $u: R_{+}^{n} \rightarrow R_{+}$be a Harnack function, $\varphi \in(0, \pi / 2)$, and $\left(b_{k}\right) \subset C(\varphi)$ a sequence such that $b_{k} \rightarrow 0, u\left(b_{k}\right) \rightarrow 0$ when $k \rightarrow \infty$. If $\left|b_{k+1}\right| \in\left(0,\left|b_{k}\right|\right)$ and there exists a number $\lambda \in(0,1)$ such that $\left|b_{k+1}\right|>\lambda\left|b_{k}\right|$ for all $k$, then $u$ has an angular limit 0 at the origin.

Proof. Fix $\psi \in(\varphi, \pi / 2)$. For $t>0$ let $A(t)=C(\psi) \cap\left(B^{n}(t) \backslash \bar{B}^{n}(\lambda t)\right)$. For $x, y \in A(t)$ we have the estimate

$$
\frac{|x-y|^{2}}{2 x_{n} \mid y_{n}} \leqq \frac{(t(1-\lambda)+2 t \sin \psi)^{2}}{2(\lambda t \cos \psi)^{2}}
$$

and hence by (2.3) $\varrho(A(t))$ has an upper bound $M$ independent of $t$. Then $C(\psi) \cap B^{n}\left(\left|b_{1}\right|\right) \subset \cup D\left(b_{k}, M\right)$, and the proof follows from Corollary 4.1.

We give examples of Harnack functions having an angular limit 0 but no limit at the origin.

4.4. Examples. (1) Define $u: R_{+}^{2} \rightarrow R_{+}$in the polar coordinates $(r, \varphi)$ by $u(r, \varphi)=r / \sin \varphi, \varphi \in(0, \pi), r>0$. After some calculation we see that $u$ satisfies (1.1) with $C_{\lambda}=((1+\lambda) /(1-\lambda))^{2}$. Clearly $u$ has an angular limit 0 at the origin, but no limit at 0 , because $u\left(1 / k, 1 / k^{2}\right) \rightarrow \infty, k \rightarrow \infty$. A corresponding bounded function (cf. $3.2(2))$ is $u /(u+1)$.

(2) Let $h:(0, \infty) \rightarrow(0, \infty)$ be an increasing continuous function with $h(t) / t \rightarrow 0$ as $t \rightarrow 0$, and let $F_{j}=\left\{\left(t, t^{j} h(t)\right): t \in(0, \infty)\right\}, j=0,1,2, \ldots, F=\bigcup_{j=0}^{\infty} F_{2 j}, E=$ $\bigcup_{j=0}^{\infty} F_{2 j+1}$. The function $u_{F}: R_{+}^{2} \rightarrow R_{+}$has the following properties (cf. (3.6)): (i) $u_{F}(x) \rightarrow 0$ as $x \rightarrow 0$ and $x \in E$ and $u_{F}\left(t e_{2}\right) \rightarrow 0$ as $t \rightarrow 0$, (ii) $u_{F} \mid F=1$. To verify (i) we calculate as in the proof of Theorem 4.5 (2) below. It follows from (i) and 4.3 that $u_{F}$ has an angular limit 0 at the origin.

A Harnack function having an asymptotic value $\alpha>0$ need not have an angular limit $\alpha$ by Example 4.2, while the situation changes by Theorem 4.3 if $\alpha=0$ and the 
curve corresponding to the asymptotic value is non-tangential. In the next theorem we will show that a positive result holds in the case $\alpha=0$ even for tangential curves, under the additional assumption that the function tends fast enough towards 0 along the curve. Such an additional assumption is necessary as we see by Example 4.4 (2) or by considering $\arg z$ on a tangential curve in $R_{+}^{2}$ (e.g. $\left\{\left(t, t^{2}\right): 0<t<1\right\}$.

4.5. Theorem. Let $u: R_{+}^{n} \rightarrow R_{+} \cup\{0\}$ satisfy (1.1), let $h:(0,1) \rightarrow R_{+}$be a continuous increasing function with $\lim _{t \rightarrow 0} h(t) / t=0$, let $M(t)=u\left(t e_{1}+h(t) e_{n}\right)$ and assume $M(t) \rightarrow 0$. (1) If $\lim M(t) h(t)^{-\varkappa}=0$, where $x=\left(\log C_{\lambda}\right) / \log ((1+\lambda) /(1-\lambda))$, then $u$ is identically equal to zero. It is not sufficient to require that $\lim \sup M(t)$. $h(t)^{-x}<\infty$. (2) If $\lim M(t)(t / h(t))^{x}=0$, then $u$ has an angular limit 0 at the origin. It is not sufficient to require that $\lim \sup M(t)(t / h(t))^{x}<\infty$.

Proof. (1) Suppose that $u\left(x_{0}\right)>0$ for some $x_{0} \in R_{+}^{n}$. Then $u\left(e_{n}\right)>0$ by Corollary 3.4. By (2.3) we get

$$
\cosh \varrho\left(e_{n}, t e_{1}+h(t) e_{n}\right)=1+\frac{t^{2}+(1-h(t))^{2}}{2 h(t)}
$$

and hence $\varrho\left(e_{n}, t e_{1}+h(t) e_{n}\right) \leqq \log (3 / h(t))$ for sufficiently small $t$. By virtue of Corollary 3.4 we get the estimate

$$
M(t) \geqq u\left(e_{n}\right) C_{\lambda}^{-1-d \log (3 / h(t))}=A u\left(e_{n}\right) h(t)^{x},
$$

where $x=\left(\log C_{\lambda}\right) / \log ((1+\lambda) /(1-\lambda)), d=1 / \log ((1+\lambda) /(1-\lambda))$, and $A=C_{\lambda}^{-1-d \log 3}$. Letting $t \rightarrow 0$ yields a contradiction. The function $u(x)=x_{n}^{*}(\neq 0)$ satisfies (1.1) with the constant $C_{\lambda}$ (cf. Example 3.1 (2) and $M(t) h(t)^{-x}=1, t \in(0,1)$. Hence $\lim \sup M(t) h(t)^{-\varkappa}<\infty$ is not sufficient.

(2) For $t>0$ write $\mu(t)=\left(t^{2}+h(t)^{2}\right)^{1 / 2}$. Then by $(2.4)$

$$
\varrho\left(\mu(t) e_{n}, t e_{1}+h(t) e_{n}\right)=-\log \tan \frac{\varphi}{2} \leqq \log (3 / \tan \varphi)
$$

where $\tan \varphi=h(t) / t$, and the inequality holds for $\varphi \in(0, \pi / 3)$. From this upper bound we get by Corollary 3.4 for small $t$

$$
u\left(\mu(t) e_{n}\right) \leqq M(t) C_{\lambda}^{1+d \log (3 t / h(t))} ; \quad d=1 / \log \frac{1+i}{1-\lambda} .
$$

By Theorem 4.3, $u$ has an angular limit 0 at the origin if $\lim u\left(\mu(t) e_{n}\right)=0$, i.e., if

$$
\lim M(t)(t / h(t))^{x}=0
$$

which is fulfilled by the assumption. The function $u_{F}(x)=\exp (-x \varrho(F, x)), F=$ $\left\{t e_{n}: t>0\right\}, x=\left(\log C_{\lambda}\right) / \log ((1+\lambda) /(1-\lambda))$, satisfies (1.1) with the constant $C_{\lambda}$ 
by Lemma 3.8. Calculating as above we see that $M(t)=u_{F}\left(t e_{1}+h(t) e_{n}\right) \rightarrow 0$ and

$$
M(t) \leqq \exp \left(-\varkappa \log \frac{t}{h(t)}\right)=\left(\frac{h(t)}{t}\right)^{x}
$$

and hence $\lim \sup M(t)(t / h(t))^{\varkappa} \leqq 1$ but $u_{F}$ fails to have an angular limit at the origin.

Suppose that $u: R_{+}^{n} \rightarrow R$ is a continuous function having an angular limit $\alpha$ at 0 . It follows from the definition of an angular limit that there exist numbers $r_{1}>r_{2}>\ldots$ $\lim r_{k}=0$ such that if

$$
E=\bigcup_{k=1}^{\infty}\left(C\left(\frac{\pi k}{2 k+1}\right) \cap B^{n}\left(r_{k}\right)\right),
$$

then $u(x) \rightarrow \alpha$ as $x \rightarrow 0, x \in E$. Clearly $E$ is tangential. It should be observed that the shape of $E$ or the order of contact between $\partial R_{+}^{n}$ and $E$ can be quite arbitrary otherwise. This fact follows from Example 4.4 (2) when $\alpha=0$, and similar examples with $\alpha>0$ can be easily constructed.

Theorem 4.5 was proved under the assumption that the function in question has an asymptotic value. It is easy to construct examples of Harnack functions having no asymptotic values whatsoever.

4.7. Example. Let $\emptyset \neq K \subset \partial R_{+}^{n}$ be a compact set nowhere dense. There existst a Harnack function having no limit at the points of $K$ and having limit 0 at $\partial R_{+}^{n} \backslash K$. The function $u_{F}: R_{+}^{n} \rightarrow R_{+}, F=K \times(0,1] \subset R_{+}^{n}$ has the asserted properties.

4.8. Example. There exists a bounded Harnack function of $R_{+}^{2}$ having no angular limit but having asymptotic value 0 at every point in $\partial R_{+}^{2}$ and having no other asymptotic values.

Let $A_{j}^{p}=\left\{x \in R: j 2^{-p} \leqq x \leqq(j+1) 2^{-p}\right\}, p \in \mathrm{N}, j=0,1, \ldots, p$ and construct $F_{p}=$ $\bigcup_{j=-\infty}^{\infty} F_{j}^{p}$ as follows for $p \geqq 2$. Set

$$
\begin{gathered}
F_{j}^{p}=A_{j}^{p} \times\left\{2^{-p j-p^{3}}\right\}, \quad j=0,1, \ldots, p \\
F_{m}^{p}=F_{j}^{p}+\frac{p+1}{2^{p}} a e_{1} \quad \text { if } \quad m=a p+j, \quad 0 \leqq j \leqq p, m \in Z .
\end{gathered}
$$

The set $F_{p}$ has the following properties.

(1) $F_{p} \subset R \times\left[2^{-p^{2}-p^{3}}, 2^{-p^{3}}\right], F_{p} \rightarrow \partial R_{+}^{2}$ as $p \rightarrow \infty$.

(2) $\varrho\left(F_{p}, F_{p+1}\right) \rightarrow \infty$.

(3) The projection of $F_{p}$ on $R$ equals $R$.

(4) The euclidean length of each component of $F_{p}$ is $2^{-p}$.

(5) $\inf _{i \neq j} \varrho\left(F_{i}^{p}, F_{j}^{p}\right) \rightarrow \infty$ as $p \rightarrow \infty$.

Let $F=\bigcup_{p=1}^{\infty} F_{p}$. The function $u_{F}: R_{+}^{2} \rightarrow R_{+}$has no angular limit by (3), but has an asymptotic value 0 at every point of $\partial R_{+}^{n}$, by (4), (5), and (2), and finally has no other asymptotic values by (2) and (1). 
We shall next study isolated singularities of Harnack functions. Let us first consider some examples.

4.9. Examples. (1) There exists a Harnack function $u: R^{n} \backslash\{0\} \rightarrow R_{+}$having no asymptotic values at the origin. Write $F=\cup_{j=1}^{\infty} S^{n-1}\left(2^{-j}\right)$. The desired function is

$$
u(x)=\exp (-k(F, x))
$$

where $k=k_{R^{n} \backslash\{0\}}$ is the quasihyperbolic metric of $R^{n} \backslash\{0\}$.

(2) The function $u: R^{2} \backslash\{0\} \rightarrow R_{+}, u(r, \varphi)=1+\sin ^{2} \varphi$ satisfies (1.1) with $C_{\lambda}=1+2 \lambda \sqrt{1-\lambda^{2}}$ and has infinitely many distinct asymptotic values at 0 .

4.10. Lemma. Let $u: R^{n} \backslash\{0\} \rightarrow R_{+}$be a Harnack function having an asymptotic value $\alpha$ at 0 . If $\alpha>0$, then there exists a number $A>0$ such that $\alpha / A \leqq u(x) \leqq \alpha A$ when $x$ is sufficiently small. If $\alpha=0$, then $u$ has limit 0 at 0 .

Proof. It follows from 2.8 (2) that $k_{R^{n} \backslash\{0\}}(x, y) \leqq \pi$ when $|x|=|y|>0$. Hence we get by Corollary 3.4

$$
u(y) C_{\lambda}^{-1-\pi / T} \leqq u(x) \leqq u(y) C_{\lambda}^{1+\pi / T} ; \quad T=\log \frac{1+3 \lambda}{1+\lambda}
$$

for $|x|=|y|>0$. The proof follows when we choose $y$ to be on the curve corresponding to the asymptotic value and let $|y| \rightarrow 0$.

4.11. Remark. The boundary behavior of monotone $\mathrm{ACL}^{n}$ functions with a finite Dirichlet integral is different from the behavior of Harnack functions (cf. [19]). For instance the functions in 4.2 and 4.9 (2) have infinite Dirichlet integrals, a fact, which follows from the results of [19] or can verified by direct calculation.

\section{Uniform continuity and uniform Harnack inequality}

In this section we will discuss a uniform Harnack inequality and characterize the functions satisfying the uniform Harnack inequality by means of uniform continuity.

5.1. The hyperbolic metric $\sigma$ in $R_{+}$. In $R_{+}$we define the hyperbolic metric $\sigma$ by the element of length $d \sigma=d x / x$. Then $\sigma(x, y)=|\log (x / y)|$ for $x, y \in R_{+}$. The symbol $\varrho$ is reserved for the hyperbolic metric in $R_{+}^{n}, n \geqq 2$.

5.2. Uniform Harnack inequality. A continuous function $u: G \rightarrow R_{+} \cup\{0\}$ is said to satisfy a uniform Harnack inequality if it satisfies the Harnack inequality (1.1) for all $\lambda \in(0,1)$ and if $C_{\lambda} \rightarrow 1$ when $\lambda \rightarrow 0$.

5.3. Examples. (1) The functions listed in 3.1 (2)-(4) and the function $u_{F}$ in Lemma 3.8 satisfy 5.2. Also the extremals of some variational integrals satisfy 5.2 ([9]). 
(2) Define $u: R_{+}^{2} \rightarrow R_{+}$by $u(x, y)=1$ for $0<y \leqq|x|^{2 / 3}, u(0, y)=2$ for $y>0$ and define $u$ elsewhere in such a way that it is continuous in $R_{+}^{2}$ and $u R_{+}^{2}=[1,2]$. Then $u$ is a Harnack function by 3.1 (1) but fails to satisfy 5.2. Note that $\lim _{r \rightarrow 0} u(r, \varphi)=0$ for $\varphi \in(0, \pi) \backslash\{\pi / 2\},=1$ for $\varphi=\pi / 2$. It follows from the requirement $C_{\lambda} \rightarrow 1$ in 5.2 that this kind of discontinuity cannot occur if 5.2 is satisfied.

5.4. Theorem. The following conditions are equivalent.

(1) $u: G \rightarrow R_{+}$satisfies 5.2 .

(2) $u:\left(G, k_{G}\right) \rightarrow\left(R_{+}, \sigma\right)$ is uniformly continuous.

(3) $\log u:\left(G, k_{G}\right) \rightarrow(R, \mid)$ is uniformly continuous.

Proof. We prove first that (1) implies (2). Fix $x \in G$. Let $y \in B^{n}(x, d(x))$. Then 5.2 yields

$$
\sigma(u(x), u(y))=\left|\log \frac{u(x)}{u(y)}\right| \leqq \log C_{|x-y| / d(x)} .
$$

From (2.6) we get

$$
|x-y| / d(x) \leqq \exp \left\{k_{G}(x, y)\right\}-1 .
$$

Because $C_{\lambda \rightarrow 1}$ as $\lambda \rightarrow 0$ the uniform continuity follows from (5.5) and (5.6).

We now prove that (2) implies (1). Fix $\lambda \in(0,1)$. Let $x \in G$ and $B^{n}(x, r) \subset G$. For $z, y \in \bar{B}^{n}(x, \lambda r)$ we have by (2.7)

$$
k_{G}(z, y) \leqq k_{G}\left(\bar{B}^{n}(x, \lambda r)\right) \leqq 2 \log \left(1+\frac{\lambda r}{r-\lambda r}\right)=2 \log \frac{1}{1-\lambda} .
$$

Bv the uniform continuity there exists a number $D_{\lambda}, D_{\lambda} \rightarrow 0, \quad \lambda \rightarrow 0$, such that

$$
\sigma(u(z), u(y))=\left|\log \frac{u(z)}{u(y)}\right| \leqq D_{\lambda}
$$

whenever $k_{G}(z, y) \leqq 2 \log (1 /(1-\lambda)), z, y \in G$. In particular, we get by (5.7)

as desired.

$$
\max _{\bar{B}^{n}(x, \lambda r)} u(w) \leqq \exp \left(D_{\lambda}\right) \min _{\bar{B}^{n}(x, \lambda r)} u(w)
$$

It remains to be shown that (2) and (3) are equivalent. But this equivalence follows from the definition of $\sigma$. Note that $\|$ in (3) is the absolute value. The proof is complete.

We next give an example of a uniformly continuous function $u:\left(R_{+}^{2}, \varrho\right) \rightarrow$ $\left(R_{+},||\right)$. Such functions will be studied in Section 6.

5.8. Example. Construction of a uniformly continuous function $u:\left(R_{+}^{2}, \varrho\right) \rightarrow$ $\left(R_{+},||\right)$, which fails to satisfy the Harnack inequality (1.1).

Let $Q=[-1,1] \times[0,2] \subset \bar{R}_{+}^{2}$. Divide $Q$ into 4 equal squares by joining the midpoints of the opposite sides. Repeat the division in those resulting squares which have one side on the $x$-axis. By continuing this process we get a decomposition of $Q$ 
into squares of constant hyperbolic size. Let $Q_{j}^{k}, j=1, \ldots, 2^{k+1}$ be the squares with side length $2^{-k}, k=0,1, \ldots$.

Let $H=\left\{(x, y, z) \in R^{3}: z=y / 10\right\}$ and $p: R^{3} \rightarrow R^{3}$ be the projection $p(x, y, z)=$ $(x, y, 0)$. We identify $R^{2}=\left\{(x, y, 0) \in R^{3}\right\}$. Let $A_{j}^{k} \subset H$ be such that $p A_{j}^{k}=Q_{j}^{k}$, let $a_{j}^{k}$ be the midpoint of $A_{j}^{k}$, and let $\partial_{H}$ stand for the boundary in the topology of $H$. Set

$$
P_{j}^{k}=\bigcup_{w \in \partial_{H} A_{j}^{k}}\left[w, a_{j}^{k}+e_{3}\right] \quad \text { and } \quad S=\left(\bigcup_{k, j} P_{j}^{k}\right) \cup\left(H \backslash \bigcup_{k, j} A_{j}^{k}\right)
$$

Define $u: R_{+}^{2} \rightarrow R_{+}$by $u(x, y)=z$ if and only if $(x, y, z) \in S$. We see that $u:\left(R_{+}^{2}, \varrho\right) \rightarrow(R,||)$ is a uniformly continuous function. We show that $u:\left(R_{+}^{2}, \varrho\right) \rightarrow$ $\left(R_{+}, \sigma\right)$ fails to be uniformly continuous. To this end let $L=\left\{(x, 3 x): x \in R_{+}\right\}$ and let $c_{1}, c_{2}, \ldots \in L$ be the successive points where $u \mid L$ attains a relative maximum with $\left|c_{j}\right|>\left|c_{j+1}\right|, c_{j} \rightarrow 0$, and let $d_{1}, d_{2}, \ldots \in L$ be the points where $u \mid L$ attains a relative minimum, $\left|d_{j}\right|>\left|c_{j}\right|>\left|d_{j+1}\right|$. By construction there exists $M>0$ such that $\varrho\left(c_{j}, d_{j}\right), \varrho\left(c_{j}, d_{j+1}\right) \leqq M$ for all $j$. Then $u\left(c_{j}\right) \rightarrow 1, u\left(d_{j}\right) \rightarrow 0$ and hence

$$
\sigma\left(u\left(c_{j}\right), u\left(d_{j}\right)\right)=\left|\log \left(u\left(c_{j}\right) / u\left(d_{j}\right)\right)\right| \rightarrow \infty .
$$

Thus $u:\left(R_{+}^{2}, \varrho\right) \rightarrow\left(R_{+}, \sigma\right)$ is not uniformly continuous, and $u$ is not a Harnack function, as desired. This example also shows that the hypothesis " $u$ is a Harnack function" in 4.1 and 4.3 cannot be replaced by the hypothesis " $u:\left(R_{+}^{n}, Q\right) \rightarrow(R,||)$ is uniformly continuous".

5.9. Remark. Let $u: G \rightarrow R$ be continuously differentiable in $G$. (1) If

$$
\sup _{y \in G}\{|\nabla u(y)| d(y)\}=M<\infty,
$$

then $u:\left(G, k_{G}\right) \rightarrow(R, \|)$ is uniformly continuous.

(2) If $u G \subset(0, \infty)$ and

$$
\sup _{y \in G}\left\{\frac{|\nabla u(y)|}{u(y)} d(y)\right\}<\infty
$$

then $u$ satisfies the uniform Harnack inequality.

Proof. (1) Let $x, y \in G$ with $k_{G}(x, y)<\log (3 / 2)$. Then $y \in B^{n}(x, d(x) / 2)$ by (2.6). By (2.6) we gef for some $\xi \in[x, y]$

$$
\begin{gathered}
|u(x)-u(y)| \leqq|\nabla u(\xi)||x-y| \leqq 2|\nabla u(\xi)| \frac{d(\xi)}{d(x)}|x-y| \\
\leqq 2 M \frac{|x-y|}{d(x)} \leqq 2 M\left(\exp \left(k_{G}(x, y)\right)-1\right),
\end{gathered}
$$

from which the assertion follows.

(2) The condition (1) for $v=\log u$ assumes the above form. The proof follows from 5.4 and (1). 


\section{Approximate limits and angular limits}

The implication' asymptotic value implies angular limit' fails to hold for functions satisfying the uniform Harnack inequality (cf. 4.2) while it holds for many other classes of functions. Therefore it is perhaps justified to consider some other properties of a function than the property of having an asymptotic value which do imply angular limit. To that end we shall consider the notion of an essential value and of an approximate limit in this section.

For the results in this section it is important that the functions are uniformly continuous with respect to the hyperbolic metric. Uniformly continuous functions have been extensively studied in complex analysis. An analytic function $f:\left(B^{2}, \varrho\right) \rightarrow$ $\left(R^{2}, \mid\right)$ is uniformly continuous if and only if it is a Bloch function [14, p. 268]. A meromorphic function $f:\left(B^{2}, \varrho\right) \rightarrow\left(\bar{R}^{2}, q\right)$, where $q$ is the spherical metric, is uniformly continuous if and only if it is a normal function [12].

6.1. Approximate limits. Let $G \subset R^{n}$ be a domain, $G \neq R^{n}$, and let $f: G \rightarrow X$ be a measurable function, where $(X, d)$ is a metric space. The function $f$ is said to have an approximate limit $\alpha$ at $x_{0} \in \bar{G} \backslash\{\infty\}$ if

$$
\lim _{r \rightarrow 0} m\left(\left(G \backslash E_{\varepsilon}\right) \cap B^{n}\left(x_{0}, r\right)\right) r^{-n}=0
$$

for every $\varepsilon>0$, where $E_{\varepsilon}=\{x \in G: d(f(x), \alpha)<\varepsilon\}$.

6.3. Measure densities. Let $E \subset R^{n}$ be a measurable set and $x \in R^{n}$. The upper measure density of $E$ at $x$ is defined to be

$$
\theta^{n *}(E, x)=\limsup _{r \rightarrow 0} m\left(E \cap \bar{B}^{n}(x, r)\right)\left(\Omega_{n} r^{n}\right)^{-1},
$$

where $\Omega_{n}=m\left(B^{n}\right)$ and the lower measure density $\theta_{n}^{*}(E, x)$ is the corresponding lim inf. If $\theta^{n *}(E, x)=\theta_{*}^{n}(E, x)$, this common value is the measure density $\theta^{n}(E, x)$ of $E$ at $x$.

The next lemma is a standard result from measure theory. It was presented without proof in [16]. A proof will be supplied here, because the result will be applied in what follows and the author has not been able to find an adequate reference to the literature.

6.4. Lemma. A measurable function $f: G \rightarrow X$ has an approximate limit $\alpha$ at $x_{0} \in \bar{G} \backslash\{\infty\}$ if and only if there exists a measurable set $E \subset G$ such that $\theta^{n}\left(G \backslash E, x_{0}\right)=0$ and $f(x)$ approaches $\alpha$ as $x$ approaches $x_{0}$ through $E$.

Proof. The sufficiency part is obvious. To prove the necessity assume that $f$ has an approximate limit $\alpha$ at $x_{0}$ and set $E_{k}=\{x \in G: d(f(x), \alpha)<1 / k\}, k=1,2, \ldots$. Then there exists $r_{k} \in(0,1)$ such that

$$
m\left(\left(G \backslash E_{k}\right) \cap \bar{B}^{n}\left(x_{0}, r\right)\right)<\frac{1}{2 k} r^{n}
$$


for $r \in\left(0, r_{k}\right]$ and $r_{k+1}^{n}<r_{k}^{n} /(2 k), k=1,2, \ldots$ Let

$$
E_{k}^{*}=\left(\bar{B}^{n}\left(x_{0}, r_{k}\right) \backslash B^{n}\left(x_{0}, r_{k+2}\right)\right) \cap E_{k}
$$

and $E=\cup E_{k}^{*}$. Then $E$ is measurable and $f(x) \rightarrow \alpha$ as $x \rightarrow x_{0}, x \in E$. For $r \in\left(r_{k+1}, r_{k}\right]$ we have

$$
\begin{gathered}
m\left((G \backslash E) \cap \bar{B}^{n}\left(x_{0}, r\right)\right) \leqq m\left(\left(G \backslash E_{k}^{*}\right) \cap \bar{B}^{n}\left(x_{0}, r\right)\right) \\
\leqq m\left(\left(G \backslash E_{k}\right) \cap \bar{B}^{n}\left(x_{0}, r\right)\right)+m\left(\bar{B}^{n}\left(x_{0}, r_{k+2}\right)\right)<\frac{1}{2 k} r^{n}+\frac{1}{2 k} r_{k+1}^{n}<\frac{1}{k} r^{n} .
\end{gathered}
$$

It follows from these estimates that $\theta^{n}\left(G \backslash E, x_{0}\right)=0$.

6.5. Notation. If $A \subset R^{n}, x \in R^{n}$, and $r>0$, then we will employ the abbreviation $A(x, r)=\bar{B}^{n}(x, r) \cap A$, which will be used in cases giving rise to no ambiguities.

6.6. Essential values. Let $f: G \rightarrow Y$ be a locally integrable function, where $Y=R^{n}$ or $=R$, and let $x_{0} \in \bar{G} \backslash\{\infty\}$ and $\alpha \in Y$. Then $f$ is said to have an essential value $\alpha$ at $x_{0}$ if

$$
\lim _{r \rightarrow 0} r^{-n} \int_{G\left(x_{0}, r\right)}|f(x)-\alpha| d m=0
$$

6.7. Remarks. (1) It follows from (6.2) that a function having an essential value has an approximate limit as well.

(2) Let $E=\left\{(x, y) \in R_{+}^{2}: y<x^{2}\right\}$ and $f(x)=(1 /|x|) \chi_{E}(x)$, where $\chi_{E}$ is the characteristic function of $E$. Then $f$ is non-bounded, has an approximate limit 0 at 0 by Lemma 6.4 and fails to have an essential value 0 at 0 .

(3) Suppose that $f: G \rightarrow R^{n}$ is locally integrable, that $|f(x)| \leqq M<\infty$ for $x \in G$ and that $f$ has an approximate limit $\alpha$ at $x_{0} \in \bar{G} \backslash\{\infty\}$. We show that $f$ has an essential value $\alpha$ at $x_{0}$ as well. In fact, let $E$ be the set in Lemma 6.4. We obtain

$$
I_{r}=\int_{G\left(x_{0}, r\right)}|f(x)-\alpha| d m \leqq \int_{E\left(x_{0}, r\right)}|f(x)-\alpha| d m+M m\left((G \backslash E)\left(x_{0}, r\right)\right) .
$$

By Lemma $6.4 a(r) \rightarrow 0$ as $r \rightarrow 0$, where $a(r)=\sup \left\{|f(x)-\alpha|: x \in E\left(x_{0}, r\right)\right\}$. Hence we get

$$
r^{-n} I_{r} \leqq r^{-n} m\left(E\left(x_{0}, r\right)\right) a(r)+M r^{-n} m\left((G \backslash E)\left(x_{0}, r\right)\right) .
$$

Letting $r \rightarrow 0$ yields the desired conclusion, since $\theta^{n}\left(G \backslash E, x_{0}\right)=0$.

It follows from (4.6) that if a continuous function of $R_{+}^{n}$ has an angular limit $\alpha$ at 0 , then it has an approximate limit $\alpha$ at 0 as well.

6.8. Remark. Applying 6.4 one can easily construct continuous bounded functions having an approximate limit but no asymptotic value, and hence no angular limit, at a given boundary point. 
We shall show that for functions uniformly continuous with respect to $\varrho$, an approximate limit implies an angular limit. For this purpose the following geometrical lemma is needed.

6.9. Lemma. Let $E \subset R_{+}^{n}$ be a measurable set such that $\theta^{n}\left(R_{+}^{n} \backslash E, 0\right)=0$ and let $L$ be a line through the origin. Then there exists a sequence $\left(a_{k}\right) \in L_{+}$such that $a_{k} \rightarrow 0, \quad\left|a_{k}\right|>\left|a_{k+1}\right|>0$ and $\left|a_{k}-a_{k+1}\right| /\left|a_{k}\right| \rightarrow 0$, and a sequence $\left(b_{j}\right) \subset E$ such that $b_{j} \rightarrow 0$ and $\left|a_{j}-a_{j}\right|<\left|a_{j}-a_{j-1}\right|, \quad \varrho\left(a_{j}, b_{j}\right) \rightarrow 0, \quad \varrho\left(b_{j}, b_{j+1}\right) \rightarrow 0$.

Proof. Let $\varphi \in(0, \pi / 2)$ be the angle between $L_{+}=L \cap R_{+}^{n}$ and the $x_{n}$-axis. When $x \in L_{+}$and $\alpha \in(0,1]$ we have

$$
\begin{aligned}
\frac{m(A)}{m(B)}=\left(\frac{\alpha \cos \varphi}{2+\alpha \cos \varphi}\right)^{n} ; \quad A & =B^{n}\left(x, \alpha \frac{|x| \cos \varphi}{2}\right), \\
B & =B^{n}\left(|x|+\alpha \frac{|x| \cos \varphi}{2}\right) .
\end{aligned}
$$

For every $k=1,2, \ldots$ there exists $r_{k} \in(0,1)$ such that $|x| \leqq r_{k}$ and $x \in L_{+}$imply

$$
B^{n}\left(x, \frac{|x| \cos \varphi}{2 k}\right) \cap E \neq \emptyset
$$

since otherwise there exists by (6.10) an integer $k_{0}$ such that

$$
\theta^{n *}\left(R_{+}^{n} \backslash E, 0\right) \geqq\left(\frac{\cos \varphi}{2 k_{0}+\cos \varphi}\right)^{n}>0,
$$

which is impossible. We may assume that $r_{k+1} \in\left(0, r_{k}\right)$ and $\lim r_{k}=0$. The sequences $\left(a_{j}\right)$ and $\left(b_{j}\right)$ will be constructed as follows. Let $a_{1} \in L \cap S_{+}^{n-1}\left(r_{1}\right)$ and select $b_{1} \in E \cap B^{n}\left(a_{1},\left|a_{1}\right|(\cos \varphi) / 2\right)$ (cf. (6.11)). Suppose that $a_{1}, \ldots, a_{j} \in L_{+}$and $b_{1}, \ldots, b_{j} \in E$ have been chosen and that $r_{k+1}<\left|a_{j}\right| \leqq r_{k}$. Let $\tilde{a}_{j+1} \in L_{+}$be such that

$$
\left|\tilde{a}_{j+1}\right|\left(1+\frac{\cos \varphi}{2(k+1)}\right)=\left|a_{j}\right|\left(1-\frac{\cos \varphi}{2 k}\right) .
$$

If $\left|\tilde{a}_{j+1}\right| \leqq r_{k+1}$, set $a_{j+1}=\tilde{a}_{j+1}$ and choose

$$
b_{j+1} \in B^{n}\left(a_{j+1}, \frac{\left|a_{j+1}\right| \cos \varphi}{2(k+1)}\right) \cap E
$$

(cf. (6.11)). Otherwise $\left|\tilde{a}_{j+1}\right|>r_{k+1}$. Let $T=(\cos \varphi) /(2 k)$ and

$$
a_{j+1}=a_{j} \frac{1-T}{1+T}, b_{j+1} \in B^{n}\left(a_{j+1}, T\left|a_{j+1}\right|\right) \cap E .
$$

In view of (2.3) it is a matter of easy verification to show that the desired conditions are satisfied.

The following result can be proved in the same way. 
6.12. Corollary. Let $E \subset R_{+}^{n}$ be a measurable set with $\theta^{n}\left(R_{+}^{n} \backslash E, 0\right)=0$, let $\varphi \in(0, \pi / 2)$ and let $\left(a_{k}\right) \subset C(\varphi)$ be a sequence such that $a_{k} \rightarrow 0$. Then there exists a non-tangential sequence $\left(b_{k}\right) \subset E$ with $b_{k} \rightarrow 0$, such that $\varrho\left(b_{k}, b_{k+1}\right) \rightarrow 0$ and $\varrho\left(a_{k}, \cup\left\{b_{j}\right\}\right) \rightarrow 0$. Hence $\lim _{|x| \rightarrow 0, x \in C(\varphi)} \varrho(x, E)=0$, for $\varphi \in(0, \pi / 2)$.

6.13. Theorem. Let $u: R_{+}^{n} \rightarrow R$ be a continuous function, $E \subset R_{+}^{n}$ with $\theta^{n}\left(R_{+}^{n} \backslash E, 0\right)=0$, and suppose that $u(x)$ tends to $\alpha$ as $x$ approaches 0 through the set $E$. Then $u$ has an angular limit $\alpha$ at 0 if one of the following conditions is satisfied.

(1) $u: R_{+}^{n} \rightarrow R_{+}$satisfies the uniform Harnack inequality.

(2) $u:\left(R_{+}^{n}, \varrho\right) \rightarrow(R, \|)$ is uniformly continuous.

Proof. Fix $\varphi \in(0, \pi / 2)$ and $\left(a_{k}\right) \subset C(\varphi)$ with $a_{k} \rightarrow 0$. It suffices to show that $u\left(a_{k}\right) \rightarrow \alpha$. The proof of (2) follows from 6.12 and the definition of uniform continuity. For (1) apply 5.4 and part (2).

6.14. Remarks. (1) Condition (1) cannot be replaced by the requirement that $u$ satisfies (1.1) as Example 5.3 (2) shows. See, however, 6.16.

(2) The condition $\theta^{n}\left(R_{+}^{n} \backslash E, 0\right)=0$ cannot be replaced by $\theta_{*}^{n}\left(R_{+}^{n} \backslash E, 0\right)=0$. To this end consider a sequence $\left(t_{k}\right), t_{k+1} \in\left(0, t_{k}\right)$ with $\lim t_{k+1} / t_{k}=0$ and set $F_{k}=$ $S_{+}^{n-1}\left(t_{k}\right), F=\cup F_{k}$, observing that $\varrho\left(F_{k}, F_{k+1}\right) \rightarrow \infty$ by (2.3). It follows (cf. 3.6) that $\quad u_{F}(x) \rightarrow 0, \quad x \rightarrow 0, \quad x \in \cup E_{k}, \quad E_{k}=\left\{x \in R_{+}^{n}:\left(t_{k} t_{k+1}^{2}\right)^{1 / 2} \leqq|x| \leqq\left(t_{k}^{2} t_{k+1}\right)^{1 / 2}\right\} \quad$ and $\theta_{*}^{n}\left(R_{+}^{n} \backslash \cup E_{k}, 0\right)=0$. Clearly $u_{F}$ has no asymptotic value, and hence no angular limit at 0 .

(3) It is not difficult to show that a bounded function $u: R_{+}^{n} \rightarrow R$ satisfying the uniform Harnack inequality 5.2 is uniformly continuous as a mapping $u:\left(R_{+}^{n}, Q\right) \rightarrow$ $(R, \|)$. For unbounded functions satisfying 5.2 such a conclusion does not hold as the example $u(x)=\exp \varrho\left(e_{n}, x\right), x \in R_{+}^{n}$, shows. See also 5.8.

It follows from Lemma 6.4 and Remark 6.7 that if $u$ satisfies (1) or (2) in 6.13 and has an essential value or approximate limit $\alpha$ at 0 , then $u$ has an angular limit as well. Results of this type are known for many different classes of functions; see for instance Diederich [4] and Mikljukov [13].

As pointed out above, Theorem 6.13 does not hold for Harnack functions in the stated generality. We shall prove a positive result in the special case $\alpha=0$. To this end, the following lemma is needed.

6.15. Lemma. Let $E \subset R_{+}^{n}$ be a measurable set such that $\theta_{*}^{n}(E, 0) \geqq \delta>0$. Then there exists a non-tangential sequence $\left(b_{k}\right) \subset E$ such that $b_{k} \rightarrow 0$ and $\lim \sup \varrho\left(b_{k}, b_{k+1}\right)<\infty$.

Proof. Fix $\varphi \in(0, \pi / 2)$ such that $m\left(B_{+}^{n} \backslash C(\varphi)\right) \leqq \Omega_{n} \delta / 4$ and $\lambda \in(0,1)$ such that $\lambda^{n} \leqq \delta / 4$. Let $r_{0} \in(0,1)$ be such that $m\left(E \cap B^{n}(r)\right) /\left(\Omega_{n} r^{n}\right) \geqq 3 \delta / 4$ for $r \in\left(0, r_{0}\right]$. For each $r \in\left(0, r_{0}\right]$ there exists $b_{r} \in E \cap(R(r, \lambda r) \cap C(\varphi))$, where $R(r, \lambda r)=B^{n}(r) \backslash$ 
$\bar{B}^{n}(\lambda r)$, because otherwise for some $r \in\left(0, r_{0}\right]$

$$
\begin{gathered}
\left(\Omega_{n} r^{n}\right)^{-1} m\left(E \cap B^{n}(r)\right) \leqq\left(\Omega_{n} r^{n}\right)^{-1} m\left(\left(B_{+}^{n}(r) \backslash \overline{C(\varphi)}\right) \cup \bar{B}_{+}^{n}(\lambda r)\right) \\
\leqq\left(\Omega_{n} r^{n}\right)^{-1}\left(\Omega_{n} \delta r^{n} / 4+\Omega_{n}(\lambda r)^{n} / 2\right) \leqq \delta / 4+\lambda^{n} / 2<\delta / 2,
\end{gathered}
$$

which is impossible. The sequence $\left(b_{j}\right)$ is constructed as follows. Set $b_{1}=b_{r_{0}}, b_{j+1}=$ $b_{\left|b_{j}\right|}$. As in the proof of Theorem 4.3 we see that there exists a number $M(\delta, n)$ such that $\varrho\left(b_{j}, b_{j+1}\right) \leqq M(\delta, n)$ for all $j=1,2, \ldots$

6.16. Corollary. Let $u: R_{+}^{n} \rightarrow R_{+}$be a Harnack function having a limit 0 at the origin through a set $E \subset R_{+}^{n}$ with $\theta_{*}^{n}(E, 0)>0$. Then $u$ has an angular limit 0 at the origin.

Proof. The proof follows from 4.3 and 6.15 .

Corollary 6.16 fails to hold for uniformly continuous functions as the following example shows.

6.17. Example. There exists a uniformly continuous $u:\left(R_{+}^{2}, \varrho\right) \rightarrow(R, \|)$ having a limit 0 through a set $E \subset R_{+}^{2}$ with $\theta_{*}^{2}(E, 0)>0$ but no angular limit. Define $u(0, y)=y / 10+1, u(x, y)=y / 10$ for $y \in(0,2|x|)$. Elsewhere $u$ is defined in such a way that $u:\left(R_{+}^{2}, \varrho\right) \rightarrow(R,||)$ is uniformly continuous (cf. 5.9 (1)). Then $u(x)$ approaches 0 through $E=\{(x, y): 0<y<2|x|\}, \theta_{*}^{2}(E, 0)>0$, but $u$ has no angular limit.

\section{References}

[1] Beardon, A. F.: Montel's theorem for subharmonic functions and solutions of partial differential equations. - Proc. Cambridge Philos. Soc. 69, 1971, 123-150.

[2] BeArdon, A. F.: The geometry of discrete groups. - Graduate Texts in Mathematics XX, Springer-Verlag, Berlin-Heidelberg-New York, 1982.

[3] Beleckaja, L. Ju., and N. S. LANdKof: Some estimates of the Harnack metric. - Izv. SeveroKavkaz. Naučn. Centra Vyš̌. Školy Ser. Estestv. Nauk. 1978, no. 2, 3-5, 116 (Russian).

[4] Diederich, J. R.: Natural limits for harmonic and superharmonic functions. - Trans. Amer. Math. Soc. 224, 1976, 381-397.

[5] Gehring, F. W., and B. G. OsGood: Uniform domains and the quasihyperbolic metric. J. Analyse Math. 36, 1979, 50-74.

[6] Gehring, F. W., and B. P. Palka: Quasiconformally homogeneous domains. - J. Analyse Math. 30, 1976, 172-199.

[7] Gilbarg, D., and N. S. Trudinger: Elliptic partial differential equations of second order. Die Grundlehren der mathematischen Wissenschaften 224, Springer-Verlag, Berlin-Heidelberg-New York, 1977.

[8] Gold'šmidt, A. I., and G. D. Suvorov: Limit sets of transformations and non-metrizable bicompactification of metric spaces. - Sibirsk. Mat. Ž. 17, no. 1, 1976, 58-74, 238 (Russian).

[9] Granlund, S., P. Lindevist, and O. Martio: Conformally invariant variational integrals. To appear. 
[10] KellogG, O. D.: Foundations of potential theory. - Dover Publications Inc., New York, 1953.

[11] KöHN, J.: Die Harnacksche Metrik in der Theorie der harmonischen Funktionen. - Math. Z. 91, 1966, 50-64.

[12] Lehto, O., and K. I. ViRTANEN: Boundary behaviour and normal meromorphic functions. Acta Math. 97, 1957, 47-65.

[13] Miksjukov, V. M.: A certain boundary property of $n$-dimensional mappings with bounded distortion. - Mat. Zametki 11, 1972, 159-164 (Russian).

[14] Pommerenke, Сh.: Univalent functions. - Vandenhoeck \& Ruprecht, Göttingen, 1975.

[15] ReŠETNJAK, JU. G.: Spatial mappings with bounded distortion. - Izdatel'stvo Nauka, Novosibirsk, 1982, (Russian).

[16] TukiA, P., and J. VÄISÄLÄ: Lipschitz and quasiconformal approximation and extension. Ann. Acad. Sci. Fenn. Ser. A I Math. 6, 1981, 303-342.

[17] VUORINEN, M.: On the existence of angular limits of $n$-dimensional quasiconformal mappings. - Ark. Mat. 18: 2, 1980, 157-180.

[18] Vuorinen, M.: Capacity densities and angular limits of quasiregular mappings. - Trans. Amer. Math. Soc. 263, 1981, 343-354.

[19] VUorinen, M.: On functions with a finite or locally finite Dirichlet integral. - In preparation.

University of Helsinki

Department of Mathematics

SF-00100 Helsinki 10

Finland

Received 1 March 1982 